\section{Kortikale Atrophie I: spezifische Signatur für Depression und geistige Defizite bei MS}

B ei MS-Patienten kommt es durch den Untergang von Nervenzellen schon sehr früh zu einem Verlust der grauen Substanz. Je nach Lokalisation kann die kortikale Atrophie Auswirkungen auf die Kognition und das Verhalten haben.

Häufig messen Forscher die Atrophie mittels voxelbasierter Morphometrie, allerdings lässt sich damit nicht zuverlässig eruieren, ob ein Volumenrückgang tatsächlich auf einer geringeren Nervenzelldichte oder auf anderen Prozessen beruht. Neurologen um Dr. Paolo Preziosa von der Universität in Mailand sind daher einen anderen Weg gegangen: Sie haben mit einem hochauflösenden 3-Tesla-MRT die Kortexdicke von MSPatienten mit und ohne kognitive Defizite oder Depressionen sowie gesunden Probanden vermessen.

An der Studie beteiligten sich 126 MSPatienten und 59 gesunde Probanden. Das Alter lag im Schnitt bei 27 Jahren. Die MS-Patienten waren im Mittel seit elf bis 13 Jahren erkrankt und hatten einen EDSS-Wert zwischen 1,5 und 2,0 Punkten. Rund die Hälfte der MS-Kranken litt an Depressionen, etwa ein Viertel klagte über deutliche kognitive Probleme, 12\% waren sowohl depressiv als auch kognitiv betroffen.

Im Vergleich zu den Probanden ließ sich bei den MS-Patienten eine ausgeprägte kortikale Ausdünnung feststellen. Erkrankte mit kortikalen Problemen waren vor allem im hinteren Parietalkortex, im oberen Orbitofrontalkortex, im Cingulum sowie im vorderen Temporal- und im Entorhinalkortex betroffen. „Diese Regionen sind bei wichtigen kognitiven Funktionen wie Arbeitsgedächtnis, Wortflüssigkeit, Problemlösung und Exekutivkontrolle beteiligt", sagte Prezioso. Bei MS-Kranken mit erhaltenen kognitiven Fähigkeiten waren diese Regionen hingegen nicht übermäßig ausgedünnt. Bei den depressiven MSKranken fanden die Neurologen vor al- lem im seitlichen Orbitofrontalkortex sowie in mittleren und unteren frontalen Arealen eine übermäßige Ausdünnung - Regionen in denen häufig auch Atrophien bei Depressiven ohne MS festgestellt werden. $\mathrm{Ob}$ die Ausdünnung als Folge der MS oder unabhängig davon die Depression verursacht hat, lässt sich anhand der Bildgebungsdaten nicht feststellen. Gleichzeitig fanden die Neurologen bei diesen Patienten aber auch einen dickeren Kortex in mittleren orbitofrontalen sowie rostralen mittleren frontalen Regionen. „Diese Bereiche sind an introspektiven mentalen Aktivitäten und der Regulation von negativen Emotionen beteiligt“, sagte Preziosa. Die Befunde passen seiner Ansicht nach recht gut zu den jeweiligen Beschwerdebildern, was darauf deuten könnte, dass die kortikale Ausdünnung im Laufe der MS solche Beschwerden hervorruft.

Thomas Müller

Oral session: Cognitive neurology/neuropsychology. O2108, Preziosa P et al. 2. EAN-Kongress,

Kopenhagen, 28. -31.5.2016

\title{
Kortikale Atrophie II: Demenz durch zu viel Fett und Zucker?
}

—ine schlechte Ernährung zählt mitt_ lerweile neben wenig Bewegung und einer soziokognitiven Trägheit zu den wichtigsten beeinflussbaren Risikofaktoren für eine Demenz, wobei sich der Einfluss der Ernährung nur schwer von anderen Risiken abgrenzen lässt: $\mathrm{Oft}$ sind es ja gerade wenig gebildete und körperliche inaktive Menschen, die auf Pommes und Bratwürste setzen. Auf der anderen Seite sind hohe Blutfett- und Blutzuckerwerte nicht besonders gut für die Gefäße, und was den Gefäßen schadet, schadet auch dem Gehirn. Die Ernährung könnte also durchaus unabhängig von anderen Faktoren einen Einfluss auf das Alzheimer- und Demenzrisiko haben.

In einer Querschnittsuntersuchung an 672 kognitiv gesunden älteren Personen im mittleren Alter von 80 Jahren (Teilnehmer der Mayo Clinic Study of Aging) beleuchteten Forscher den Zusammenhang zwischen Kortexdicke und Ernährung per MRT. Insbesondere interessierte sie die Kortexdicke in solchen Arealen, die bei Alzheimer stark betroffen sind, darunter Bereiche im entorhinalen Kortex sowie im unteren und mittleren Temporallappen. Alle Teilnehmer füllten einen ausführlichen Ernährungsfragebogen aus, über den sich Fett-, Zucker-, Kalorien- und Vitaminaufnahme bestimmen ließen.

Bei Teilnehmern mit erhöhtem Kohlenhydratkonsum im Allgemeinen und mit viel Zucker im Besonderen ließ sich ein signifikant dünnerer Kortex in den Alzheimer-Regionen nachweisen als bei Teilnehmern mit ausgewogener Ernährung. Eine ähnliche Beziehung konnten Neurologen um Dr. Sarah Staubo von der Karls-Universität in Hradec Králové, Tschechien, auch bei erhöhtem Gesamt- fettanteil sowie bei vermehrtem Konsum von gesättigten Fetten feststellen. Wurden hingegen Personen untersucht, die viele Vitamine über die Nahrung aufnahmen, was auf einen hohen Obst- und Gemüsekonsum deutet, so war die Kortexdicke in den Alzheimer-Risikoregionen signifikant erhöht. Gesamtkalorien, Bildungslevel, BMI und andere vaskuläre Faktoren wie Diabetes, Hypertonie oder KHK wurden bei diesen Berechnungen berücksichtigt. Da es sich jedoch um eine Querschnittstudie mit subjektiven Angaben handelt, lässt sich nicht sagen, was Ursache und Wirkung ist. Auch könnten weitere Faktoren wie Bewegungsarmut oder soziale Isolation das Ergebnis verzerrt haben: Wer im Alter alleine lebt, ernährt sich häufig ungesünder als ältere mit guten sozialen Kontakten. Vielleicht ist hier aber eher die Isolation der relevante Schrumpffaktor.

Thomas Müller

Oral Session: Ageing and dementia. Staubo $S$ et al. O2204. 2. EAN-Kongress, Kopenhagen, 28.-31.5.2016 